\title{
Abondance d'Aspidiscus cristatus (LAMARCK, 1801) dans la Formation des Marnes de Smail de la région de Batna (NE d'Algérie) : Une espèce caractéristique pour le Cénomanien moyen
}

\author{
Sihem SALMI-LAOUAR ${ }^{1}$ \\ Bruno FerRÉ ${ }^{2}$
}

\section{Riadh AouIssI ${ }^{3}$}

\begin{abstract}
Résumé : Les marnes cénomaniennes de la région de Batna (nord-est de l'Algérie) montrent plusieurs niveaux fossilifères, à faune très diversifiée. Parmi les fossiles présents en abondance et en bon état de conservation figure Aspidiscus cristatus (LAMARCK, 1801). Cette espèce de polypier a été rattachée à la famille des Latomeandridae Alloiteau, 1952, en raison de la présence de pennulae et de perforations concentrées sur le bord interne des septes. Dans les coupes étudiées, Aspidiscus cristatus se rencontre dans des niveaux allant de la Zone à Cunningtoniceras inerme à la Zone à Acanthoceras amphibolum, avec une relative abondance dans la Zone à Acanthoceras cf. rhotomagense. Cette espèce est, par conséquent, un bon marqueur du Cénomanien moyen de la région de Batna. Nous donnons ici une description paléontologique et discutons des cadres environnemental et paléogéographique de cette espèce. En effet, la morphologie externe de son test, convexe sur la face supérieure, plane à concave sur la face inférieure, évoque une adaptation à des environnements marins de faible énergie, de substrat vaseux et au taux de sédimentation élevé. La répartition paléogéographique de cette espèce, avec des spécimens de taille souvent plus grande sur les marges sud et est du domaine téthysien que sur la marge nord, témoigne d'un gradient thermique et d'une zonation climatique spécifiques, avec des eaux relativement plus chaudes sur la marge sudtéthysienne au Cénomanien, favorisant une bio-érosion beaucoup plus fréquente et plus poussée des spécimens fossiles des régions sud-téthysiennes que celle affectant les spécimens des régions septentrionales de la Téthys.
\end{abstract}

\section{Mots-clefs :}

- Aspidiscus cristatus ;

- paléontologie :

- paléo-environnement ;

- paléogéographie :

- Cénomanien moyen ;

- Batna ;

- Algérie

Citation: Salmi-Laouar S., Ferré B. \& Aouissi R. (2019).- Abondance d'Aspidiscus cristatus (LAMARCK, 1801) dans la Formation des Marnes de Smail de la région de Batna (NE d'Algérie) : Une espèce caractéristique pour le Cénomanien moyen.- Carnets Geol., Madrid, vol. 19, no 10, p. 185-197.

Abstract: Abundant occurrence of Aspidiscus cristatus (LAMARCK, 1801) in the "Marnes de Smail" Formation from the Batna area (NE-Algeria): Index species for the Middle Cenomanian.- The Cenomanian marls of the Batna region (NE Algeria) display several fossiliferous beds with highly diversified fauna. Among the abundant and well-preserved fossils stands out Aspidiscus cristatus (LAMARCK, 1801). This coral taxon is assigned to the Family Latomeandridae AlLOITEAU, 1952, due to the presence of pennulae and inner septal margins with slight perforations. In the studied sections Aspidiscus cristatus is found in beds ranging from the Cunningtoniceras inerme Zone to the Acanthoceras amphibolum Zone, with a relative abundance within the Acanthoceras cf. rhotomagense Zone. This species is subsequently a good marker for the Middle Cenomanian in the Batna district. We

\footnotetext{
${ }^{1}$ Université Badji Mokhtar, Annaba, Laboratoire de Recherche de Géologie (LRG), B.P. 12, 23000 Annaba (Algérie) ssalmit@yahoo.fr

2 Dame du Lac 213, 3 rue Henri Barbusse, F-76300 Sotteville-lès-Rouen (France)

bruno_ferre@yahoo.fr

${ }^{3}$ Université Larbi Ben M'hidi, Oum el Bouaghi, Laboratoire de Recherche de Géologie (LRG), B.P. 12, 23000 Annaba (Algérie) aouissi_riadh@hotmail.com
}

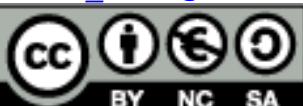

Published online in final form (pdf) on August 8, 2019

[Éditeur : Bruno GRANIER; éditeur pour la langue anglaise : Robert W. SCOTT] 
give herein a systematic and paleontological description and discuss the paleoenvironmental and paleogeographic framework of this taxon. Actually the outer morphology of its test, with convex outer surface and planar to concave inner surface, suggests an adaptation to mud-supported, low-energy marine environments with high sedimentary rate. The paleogeographic range of this taxon, with often larger-size specimens on the southern and eastern margins of the Tethysian Realm, than those on the northern margin, supports very peculiar thermal gradient and climactic zone, with relatively warmer waters on the southern Tethysian margin during the Cenomanian, favoring a more frequent and more effective bioerosion on fossil specimens from southern Tethysian areas than that affecting specimens from northern Tethysian areas.

\section{Key-words:}

- Aspidiscus cristatus;

- paleontology;

- paleoenvironment;

- paleogeography;

- Middle Cenomanian;

- Batna;

- Algeria

\section{Introduction}

Au nord de la ville de Batna (435 km au sudest de la ville d'Alger, Fig. 1A-B), des dépôts marins du Crétacé affleurent sur de larges étendues, montrant des séries cénomaniennes pouvant atteindre une épaisseur de l'ordre de $600 \mathrm{~m}$ (BUREAU, 1975). En dépit de l'abondance de ces affleurements et de leur richesse en macro-invertébrés, les travaux publiés restent rares et se limitent à des descriptions d'ordre général, lithologique (SAVORNIN, 1931; BELLION, 1972 ; ViVIËRE, 1985 ; YAHIAOUI, 1990) et parfois structural (BUREAU, 1975, 1986; VILA, 1980). Ainsi, le recensement paléontologique de la macrofaune cénomanienne reste sommaire (LAFFITE, 1939). La subdivision chrono-stratigraphique du Cénomanien établie par SLAMI et al. (2018) à Thénièt el Manchar, et celle de Aouissi et al. (2018) à Métrassi décrivent en détail les différents assemblages fauniques de la région de Batna et mettent alors en évidence un Cénomanien moyen marneux riche en Aspidiscus cristatus (LAMARCK, 1801). L'étude d'un matériel abondant de cette espèce dans les dépôts cénomaniens de la région de Batna nous a permis de préciser sa répartition stratigraphique, ses variations morphométriques (taille et forme) et de réexaminer sa position systématique. L'excellent état de conservation des traces de la bio-érosion sur les spécimens étudiés, surtout par perforation, fait de ce taxon une espèce de grand intérêt pour les interprétations paléo-environnementales des gisements péritéthysiens où ce taxon considéré comme un marqueur bio-zonal potentiel et important, au moins à l'échelle locale et régionale.

\section{Cadre géologique général}

La région de Batna (Fig. 1.A) se situe dans une zone de convergence entre les Monts de Bellezma au Nord et le domaine des Aurès au Sud (Guiraud, 1973). Les dépôts sédimentaires connus dans cette région vont du Trias au Quaternaire, avec des niveaux du Crétacé supérieur formant de larges affleurements (LAFFITE,
1939 ; Burollet, 1956 ; Bellion, 1972 ; Guiraud, 1973 ; ViLA, 1980 ; BUREAU, 1986 ; YAHIAOUI, 1990) (Fig. 1.B). La sédimentation de ces dépôts s'est effectuée aux Monts de Bellezma dans un milieu de type plate-forme, caractérisé par des faciès de milieu peu profond et peu subsident (YAHIAOUI, 1990), alors qu'il s'agit dans les régions des Aurès (HERKAT, 2005) de milieu de type bassin intracratonique plus subsident. Ces dépôts crétacés ont été structurés en plis anticlinaux de direction sensiblement NE-SW et à grand rayon de courbure, conséquence de deux phases de compression majeures: atlasique, au Jurassique, puis alpine, au Cénozoïque (BeLlion, 1972 ; BUREAU, 1975 ; YAHIAOUI, 1990).

\section{Matériel étudié}

Au total, 350 spécimens d'Aspidiscus cristatus ont été récoltés dans plusieurs gisements de la région de Batna: Thénièt El Manchar $\left(35^{\circ} 11^{\prime} 13^{\prime \prime} \mathrm{N}\right.$, $\left.4^{\circ} 00^{\prime} 00^{\prime \prime} \mathrm{E}\right)$, Métrassi $\left(35^{\circ} 40^{\prime} 0^{\prime \prime} \mathrm{N}, 4^{\circ} 9^{\prime} 0^{\prime \prime} \mathrm{E}\right)$, Bouarif $\left(35^{\circ} 36^{\prime} 50^{\prime \prime} \mathrm{N}, 6^{\circ} 26^{\prime} 22^{\prime \prime} \mathrm{E}\right)$, Thénièt el Baida $\left(36^{\circ} 01^{\prime}\right.$ $\left.12^{\prime \prime} \mathrm{N}, 3^{\circ} 36^{\prime} 00^{\prime \prime E}\right)$, Djebel Fakra $\left(36^{\circ} 03^{\prime} 00^{\prime \prime} \mathrm{N}, 3^{\circ} 34^{\prime}\right.$

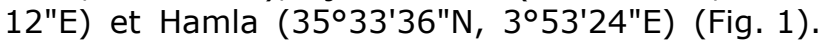
Toutefois, seuls 63 spécimens ont été étudiés pour des fins biométriques. Ce matériel fait l'objet d'un projet d'exposition des macro-invertébrés de la région de Batna déposés au Laboratoire de Recherche en Géologie (LRG) de I'Université Badji Mokhtar à Annaba.

\section{Description lithologique \\ de la Formation des Marnes de Smail}

Tous les spécimens récoltés proviennent de la partie moyenne de la Formation des Marnes de Smail. Cette formation, à large extension régionale est datée du Cénomanien (YAHIAOUI, 1990); elle est constituée d'un faciès monotone essentiellement marneux à rares minces bancs calcaires et renfermant une macrofaune assez diversifiée. Les niveaux livrant en abondance Aspidiscus cristatus se situent dans le Membre des Marnes à Aspidiscus cristatus, défini par SLAMI et al. (2018) et Aouissi et al. (2018). Ce scléractiniaire solitaire y est associé à des bivalves, des gastéropodes, des ammonites et des échinides (Fig. 2). 
L'abondance de ce polypier dans les niveaux susmentionnés et la morphologie externe des individus indiquent, pour les dépôts cénomaniens de la région étudiée, un paléo-environne- ment marin peu profond, à énergie faible, au taux de sédimentation élevé et au substrat vaseux (GILL \& CHIKHI, 1991).

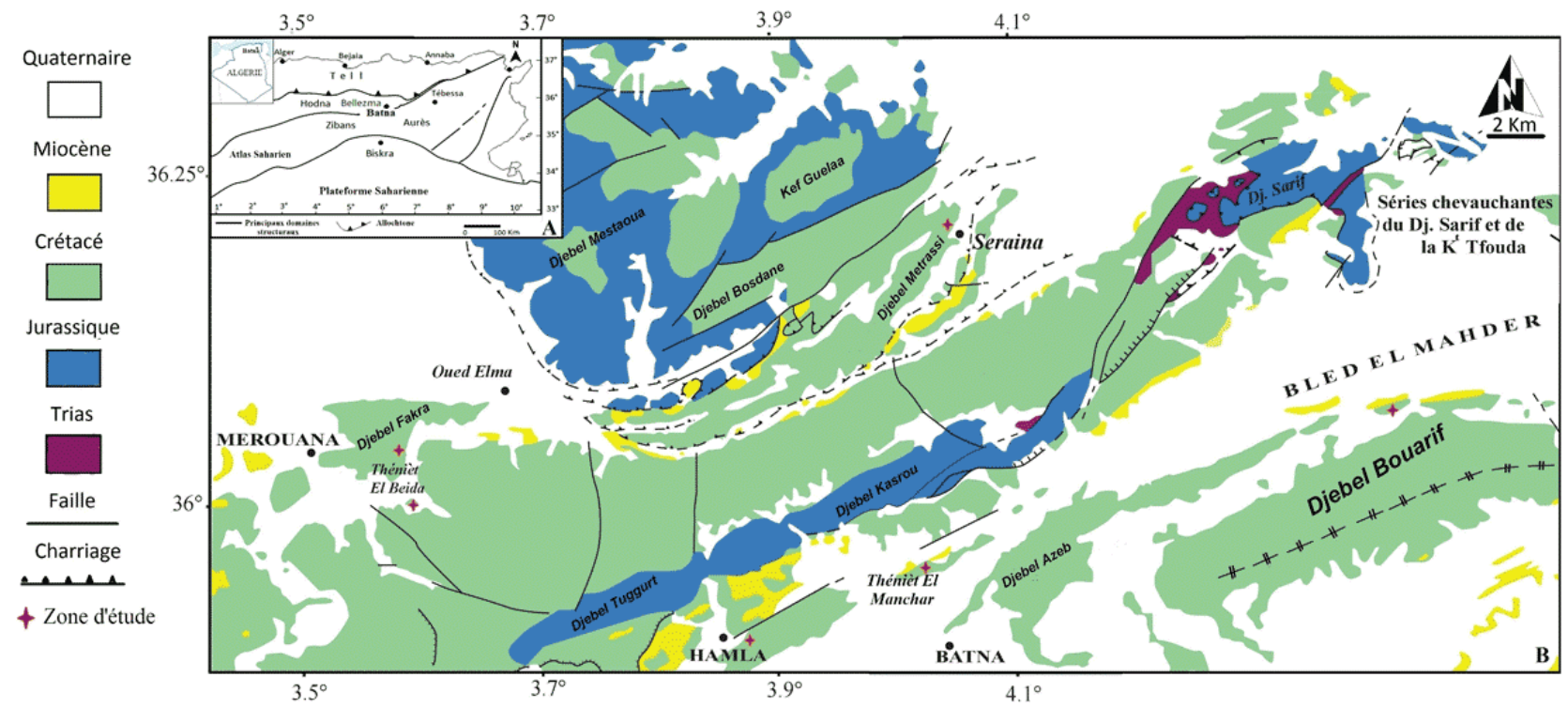

Figure 1 : A- Carte structurale simplifiée du nord de l'Algérie et localisation de la région de Batna (d'après HERKAT, 2007) ; B- Carte géologique de la région au nord de la ville de Batna (d'après la carte géologique au 1/50,000 de Merouana-El Ksar).

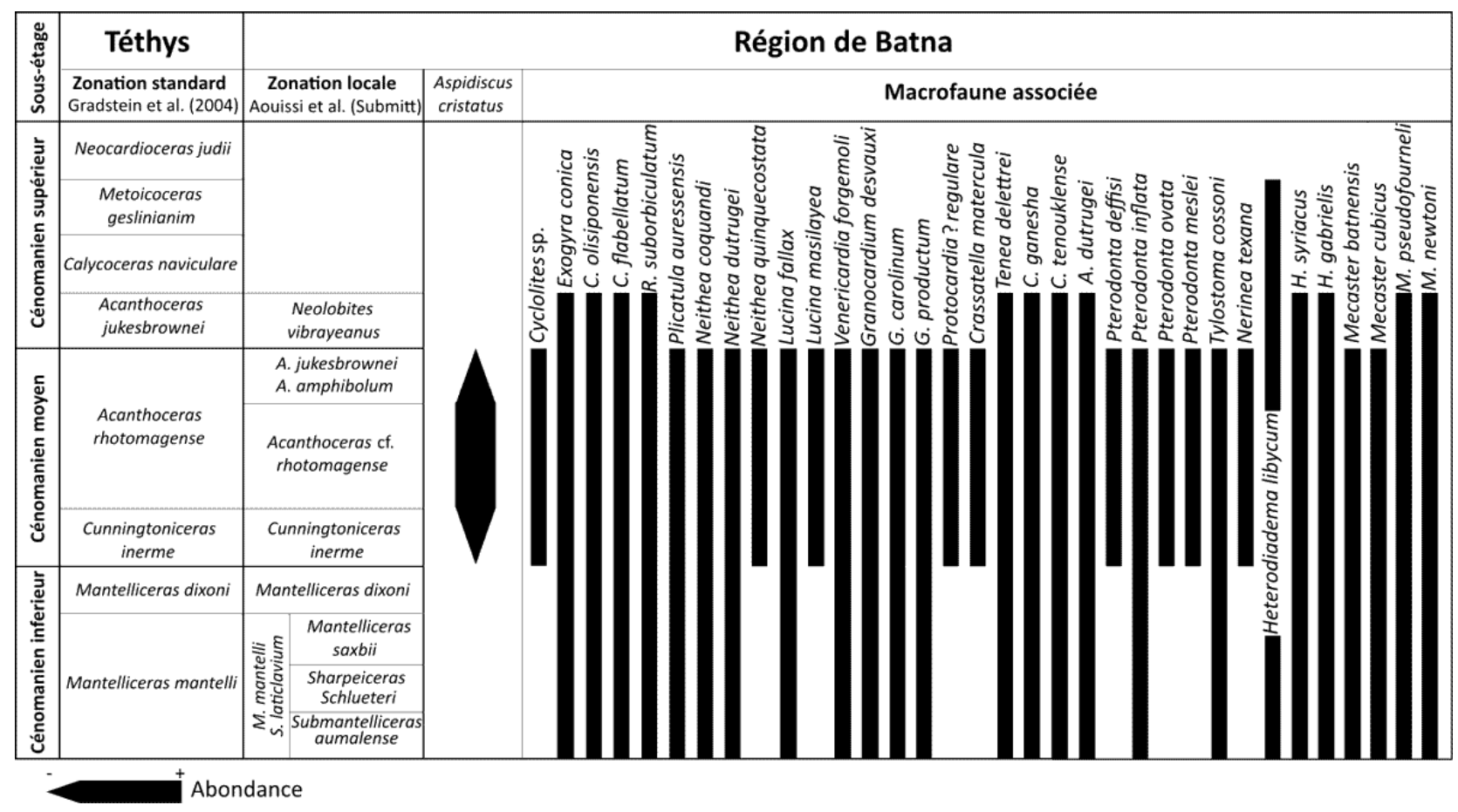

Figure 2 : Zonation standard des ammonites du domaine téthysien et de la région de Batna et répartition stratigraphique de la macrofaune (hors ammonites) dans les dépôts du Cénomanien de la région de Batna (Aouissi et al., en préparation). 


\section{Systématique \\ Ordre Microsolenina MORYCOWA \& RONIEWICZ, 1995}

\section{Famille Latomeandridae Alloiteau, 1952}

Genre Aspidiscus KönIG, 1825

\section{Aspidiscus cristatus (LAMARCK, 1801)}

\subsection{Synonymie}

1801 Cyclolites cristata sp. nov. LAMARCK, p. 369. 1991 Aspidiscus cristatus (LAMARCK, 1821) : GILL \& CHIKHI, p. 349, Figs. 1-2. (synonymie) 1995 Aspidiscus cristatus (LAMARCK, 1821) : ABDELGAWAD \& GAMEIL, p. 23, PI. 9, fig. 6.

2002 Aspidiscus cristatus (LAMARCK, 1821): BARONSZABO, p. 144, Ffig. 53.

2011 Aspidiscus cristatus (LAMARCK, 1821) : PANDEY et al., p. 31, PI. I, figs. 1-6.
2014 Aspidiscus cristatus (LAMARCK, 1821) : WiLSON et al., p. 243, Figs. 2-4.

2018 Aspidiscus cristatus (LAMARCK, 1821) : SLAMI et al., Fig. 5 d.

2018 Aspidiscus cristatus (LAMARCK, 1821) : AouISSI et al., p. 7, Fig. 4(1a-b).

\subsection{Mesures des spécimens étudiés}

Les mesures relevées sur les spécimens étudiés (Tabl. 1) sont les suivantes :

Dc : distance entre les centres de deux corallites adjacents ;

Ns : nombre de septes par $\mathrm{mm}$;

$\mathrm{H}$ : hauteur du test ;

$D$ : diamètre du test ;

$\mathrm{D} / \mathrm{H}$ : rapport diamètre sur hauteur ;

Nbr : effectif de la population.

Toutes les mesures sont données en millimètres.

Tableau 1 : Paramètres des spécimens étudiés.

\begin{tabular}{|c|c|c|c|c|c|c|c|c|c|c|c|}
\hline$N=63$ & Nbr & Étendue & $D(\mathrm{~mm})$ & $H(\mathbf{m m})$ & $D / H$ & Moyenne & $D(\mathrm{~mm})$ & $H(\mathbf{m m})$ & $D / H$ & $\begin{array}{c}\text { Ns } \\
\text { par } \mathbf{m m}\end{array}$ & $\begin{array}{c}\text { Dc } \\
(\mathrm{mm})\end{array}$ \\
\hline $\mathrm{D}$ & 18 & & $23-30$ & $9.5-13.6$ & $1.69-2.81$ & & $25.7-26.98$ & $10.65-11.29$ & $2.22-2.34$ & 6 & $0.6-1.4$ \\
\hline $\mathrm{D}$ & 32 & & $30.2-39.1$ & $10-17$ & $2.0-3.4$ & & $34.7-35.05$ & $12.53-13.17$ & $2.65-2.79$ & $6-7$ & $0.6-1.6$ \\
\hline D & 13 & & $40.1-49.3$ & $12.1-20.1$ & $2.12-3.28$ & & $43.13-44.69$ & $15.45-16.75$ & $2.66-2.9$ & 7 & $0.8-1.8$ \\
\hline
\end{tabular}

\subsection{Description paléontologique}

Polypier à contour circulaire à subcirculaire, à face supérieure convexe (PI. I, figs. 1a, 2a, $3 a, 4 a, 8 a$ ) et face inférieure plate (PI. I, figs. $1 b, 2 b, 3 b$ ) ou concave (PI. I, figs. $4 b, 5$, $8 \mathrm{~b})$. Les monticules sont disposés radialement entre les crêtes et les centres du calice sont arrangés en série le long de sillons dans un arrangement dit hydnophoroïde (PI. I, figs. 6$7)$. Les monticules ont un contour circulaire à ovale. Les crêtes, ou collines, sont distinctes et augmentent successivement en nombre vers la périphérie du test. Le centre des calices, modérément distincts, est limité par des monticules d'un côté et par une crête de l'autre côté, ou par des crêtes des deux côtés.

Une couronne périphérique distincte couverte de septes, s'étend jusqu'à la marge en angle droit, et continue en une zone étroite au-dessus de la surface inférieure des côtes (PI. I, fig. 8a).

La face inférieure est couverte d'une holothèque mince avec un arrangement concentrique des crêtes et des plis (PI. I, fig. 8b), sans zone d'attachement. La face supérieure montre une crête médiane incurvée (PI. II, fig. 1) ou sinueuse (PI. II, fig. 2), qui divise la surface à parts égales.

L'examen des différents spécimens d'Aspidiscus cristatus révèle qu'un nombre important d'entre eux sont affectés par la bio-érosion. Cette dernière se traduit par la présence de perforations et d'incrustations sur les deux faces, supérieure et inférieure (PI. II, figs. 1-7).

\section{Répartition géographique et stratigraphique}

Dans le secteur de Batna, l'essentiel des spécimens d'Aspidiscus cristatus ont été recueillis dans le Membre des Marnes à Aspidiscus cristatus de la partie médiane de la Formation des Marnes de Smail (AouISSI et al., 2018 ; SLAMI et al., 2018). La répartition stratigraphique de ce scléractiniaire établie par ces mêmes auteurs, s'inscrit depuis la Zone à Cunningtoniceras inerme, de la base du Cénomanien moyen, jusqu'à la Zone à Acanthoceras jukesbrownei et la Zone à $A$. amphibolum, du sommet du Cénomanien moyen. L'intervalle d'abondance de ce taxon correspond à la Zone à Acanthoceras cf. rhotomagense, de la partie moyenne du Cénomanien moyen. Ainsi, grâce aux assemblages d'ammonites (étudiés par Aouissi et al., en préparation), il nous est possible de considérer l'intervalle d'abondance d'Aspidiscus cristatus comme une zone marqueur du Cénomanien moyen dans la région étudiée (Fig. 2).

La présence d'Aspidiscus cristatus a été signalée dans plusieurs localités de part et d'autre de la Méditerranée (Fig. 4). D'après GILL et CHIKHI (1991), 70 de ces localités ont livré au moins une espèce du genre Aspidiscus, dont plus de 60 ont livré exclusivement Aspidiscus cristatus. La distribution géographique large de cette espèce, et son 


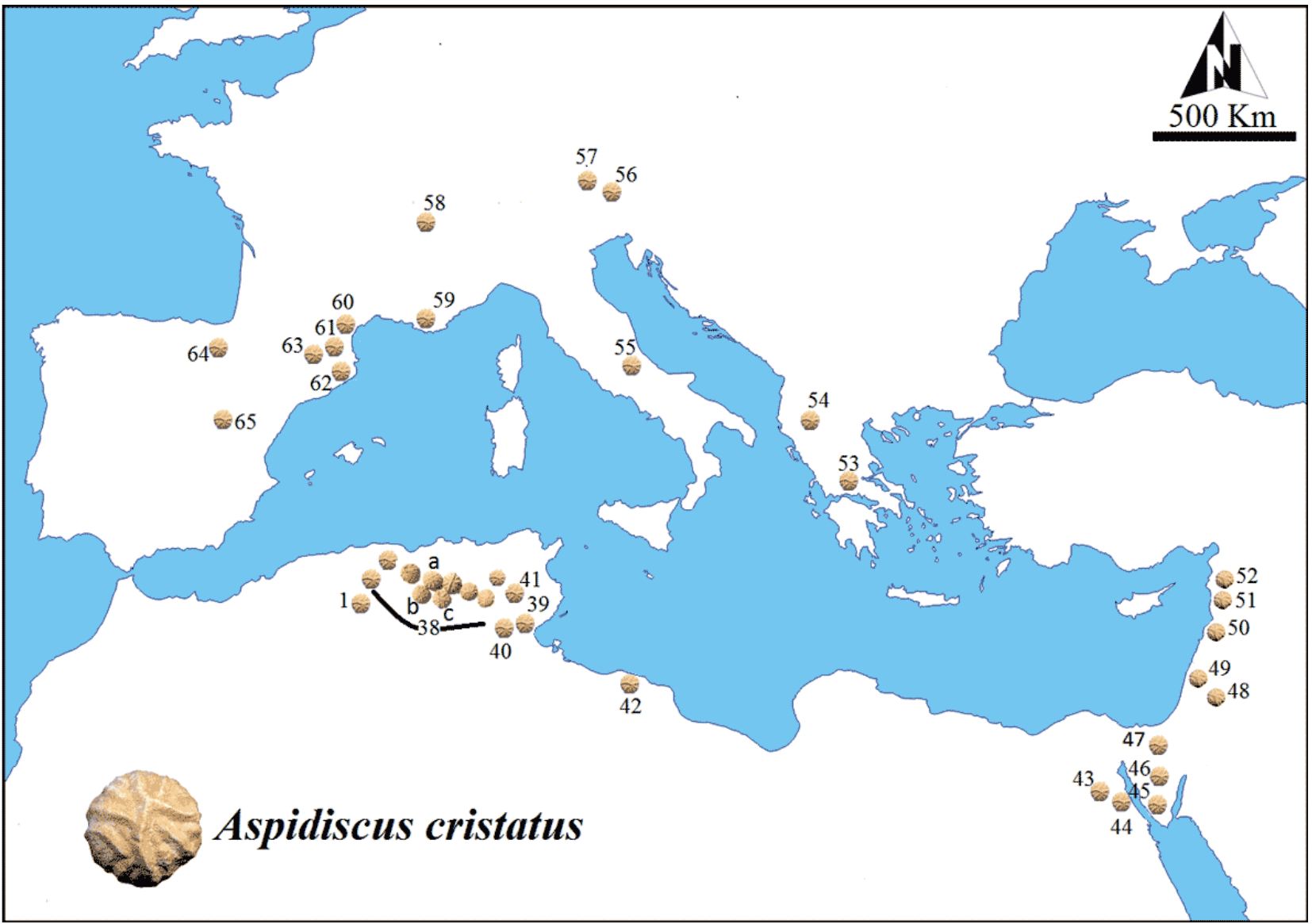

Figure 3 : Répartition géographique d'Aspidiscus cristatus dans les bassins du pourtour méditerranéen. 1-38: Algérie (EDWARDS et HAIME, 1849 ; CoQUAND, 1862 ; GILL et CHIKHI, 1991) ; a, b, c : Batna (ce travail) ; 39-41 : Sbeita, Tunisie (ShaW, 1738 ; Alloiteau, 1952) ; 42 : Tripoli, Libye (Alloiteau, 1952) ; 43-47 : Égypte (AvNimeleCH, 1947 ; Thomas et OMARA, 1957 ; ABdel-GaWAD et GAMeIL, 1995 ; PANDEY et al., 2011) ; 48 : Jordanie (Thomas et OMARA, 1957); 49 : Israël (Wilson, 2014); 50 : Liban (ThOMAS et OMARA, 1957) ; 51-52 : Syrie (ThOMAS et OMARA, 1957) ; 53 : Dremisa, Grèce (RENZ, 1930) ; 54 : Korça, Albanie (Dreger, 1892) ; 55 : Italie (DE ANGELIS D'OSSAT, 1901); 56 : Vils, Frontière germano-autrichienne (LAPPARENT, 1906) ; 57 : Ruhpolding et Urschlau, Allemagne (SÖHLE, 1897 ; SCHLOSSER, 1924) ; 58-63 : Bassin de Paris et Sud de la France (LAMOUROuX, 1821; FROMENTEL, 1877 ) ; 64-65 : Catalogne, Espagne (BARON-SZABO, 2002).

extension stratigraphique courte, en font un excellent outil de corrélation à grande distance. Ce taxon a été signalé dans les parties moyenne et supérieure du Cénomanien dans la plupart des bassins périméditerranéens (Tabl. 2), en particulier en Égypte (ABDEL-GAWAD \& GAMEIL, 1995 ; PANDEY et al., 2011 ; WILSON et al., 2014). En Algérie, l'extension stratigraphique d'Aspidiscus cristatus est limitée au Cénomanien moyen (GILL \& CHIKHI, 1991).

\section{Paléoécologie}

Contrairement à la majorité des scléractiniaires, qui nécessitait un substrat compétent pour assurer leur fixation, Aspidiscus cristatus a par contre adopté un mode de vie libre caractérisé par l'absence de trace de fixation. Cette espèce a donc privilégié des substrats mous sur lesquels ils reposaient librement. Ayoub HanNa (2011) signale la présence d'Aspidiscus cristatus dans des faciès marneux du Cénomanien et note également l'absence de cette espèce dans les formations gréseuses et calcaires du Cénomanien. En Algérie, Aspidiscus cristatus est surtout présent dans des interbancs argilo-marneux friables. Le Tableau 2 illustre les environnements sédimentaires de quelques gisements cénomaniens à Aspidiscus cristatus du domaine téthysien, qui sont similaires à ceux des gisements de l'Algérie. En effet, la stabilité des coquilles de ces organismes sur les substrats mous est assurée, d'une part, par la forme de la face inférieure, concave à plate et, d'autre part, par les plis concentriques de l'holothèque qui servent à stabiliser le test contre toute dérive latérale. La forme convexe fortement plissée de la face supérieure facilite l'évacuation des particules sédimentaires en suspension (HUBBARD, 1973 ; HUBBARD \& POCOCK, 1972 ; WiJsman-Best, 1972, 1974 ; PANDEY et al., 2011). Dans ce contexte, Aspidiscus cristatus semble fortement contraint par le type du faciès et ne tolère qu'une gamme restreinte de particules cantonnées dans des conditions de basse énergie, avec des dépôts à grains fins, aboutissant à un substrat vaseux, même avec un taux de sédimentation élevé. 
Tableau 2 : Extension stratigraphique d'Aspidiscus cristatus et faciès associés à travers quelques bassins du pourtour méditerranéen.

\begin{tabular}{|c|c|c|c|c|c|c|c|}
\hline Pays & Israël & \multicolumn{2}{|c|}{ Égypte } & Tunisie & \multicolumn{3}{|c|}{ Algérie } \\
\hline $\begin{array}{l}\text { Auteur (s) et } \\
\text { Localité }\end{array}$ & $\begin{array}{c}\text { WILSON } \\
(2014) \\
\text { Yorqe'am }\end{array}$ & $\begin{array}{l}\text { PANDEY et al. } \\
(2011) \\
\text { Sinaï }\end{array}$ & $\begin{array}{c}\text { ABDEL-GAWAD } \\
(1995) \\
\text { Sinaï }\end{array}$ & $\begin{array}{l}\text { SHAW (1738) } \\
\text { ALLOITEAU (1952) } \\
\text { Tunisie centrale }\end{array}$ & $\begin{array}{l}\text { CHIKHI et GILL } \\
(1991) \\
\text { Boussaâda }\end{array}$ & $\begin{array}{c}\text { COQUAND } \\
(1862) \\
\text { Aurès et Tébessa }\end{array}$ & présent travail \\
\hline Âge & Cénomanien & $\begin{array}{l}\text { Cénomanien } \\
\text { moyen }\end{array}$ & $\begin{array}{l}\text { Cénomanien } \\
\text { supérieur }\end{array}$ & Cénomanien & $\begin{array}{l}\text { Cénomanien } \\
\text { moyen }\end{array}$ & $\begin{array}{l}\text { Carentonien (- } \\
\text { Cénomanien } \\
\text { supérieur) }\end{array}$ & $\begin{array}{l}\text { Cénomanien } \\
\text { moyen }\end{array}$ \\
\hline Faciès & Marnes & Marnes & Marnes & Marnes & Marnes & Marnes grisâtres & $\begin{array}{l}\text { Marnes } \\
\text { argileuses }\end{array}$ \\
\hline Pays & Espagne & France & \multicolumn{2}{|c|}{ Allemagne } & Autriche & Albanie & Liban et Syrie \\
\hline $\begin{array}{c}\text { Auteur (s) et } \\
\text { Localité }\end{array}$ & $\begin{array}{c}\text { BARON-SZABO } \\
(2002) \\
\text { Catalogne }\end{array}$ & $\begin{array}{l}\text { LAMOUROUX et al. } \\
(1824) \\
\text { Sinaï }\end{array}$ & $\begin{array}{c}\text { SÖHLE } \\
(1897) \\
\text { Ruhpolding }\end{array}$ & $\begin{array}{c}\text { SCHLOSSER } \\
(1924) \\
\text { Urschlau }\end{array}$ & $\begin{array}{l}\text { LAPPARENT } \\
(1901) \\
\text { Vils }\end{array}$ & $\begin{array}{l}\text { DREGER } \\
(1892) \\
\text { Korça }\end{array}$ & $\begin{array}{c}\text { THOMAS et OMARA } \\
(1857)\end{array}$ \\
\hline Âge & Cénomanien & Cénomanien & $\begin{array}{l}\text { Cénomanien } \\
\text { avancé }\end{array}$ & Cénomanien & Cénomanien & Cénomanien & Cénomanien \\
\hline Faciès & / & $\begin{array}{l}\text { Marnes } \\
\text { bleuâtres }\end{array}$ & Marnes jaunes & / & $\begin{array}{c}\text { Marnes } \\
\text { glauconieuses }\end{array}$ & Marnes & / \\
\hline
\end{tabular}

Sur le plan nutritif, Aspidiscus cristatus est un microphage filtreur se nourrissant des particules organiques en suspension dans l'eau, en particulier les organismes planctoniques, qui chutent notamment depuis la tranche supérieure des eaux marines.

Les traces de bio-érosion, rencontrées sur les deux faces de certains spécimens, sont attribuées à l'action érosive de certains organismes épi- et endobiontes. Il s'agit généralement de minuscules bivalves qui perforent spécialement la surface supérieure, mais aussi la surface inférieure des tests de la proie. Cette perforation (PI. II, figs. 1-2, 4-6) est à rapprocher de l'ichnogenre Gastrochaenolites LEYMERIE, 1842 (KELLY \& BROMLEY, 1984). On note parfois la présence de deux perforations juxtaposées sur la face supérieure qui finissent dans une même cavité interne. Certaines perforations ont un diamètre pouvant atteindre $7 \mathrm{~mm}$. D'autres spécimens montrent des tunnels en forme de croissant (PI. II, fig. 3), qui peuvent être attribués à l'ichnogenre Maeandropolydora (VoIGT, 1975), ou des tubes agrégés longs roulés de façon aléatoire attribués à l'espèce Glomerula serpentina (GoLDFUSS, 1831).

\section{Discussion}

La forme convexe de la face supérieure, et concave de la face inférieure du test d'Aspidiscus cristatus est très caractéristique de cette espèce et rend sa détermination assez aisée. La bonne conservation des microarchitectures permet l'identification formelle de l'espèce. La présence de pennulae et de perforations concentrés sur le bord interne des septes confirme l'appartenance de cette espèce à la famille des Latomeandridae (Alloiteau, 1952).
Le rapport crête-monticule montre qu'Aspidiscus cristatus est dominé par des crêtes avec quelques monticules. En revanche, Aspidiscus felixi RENZ, 1930, comporte des monticules emballés en masse mais dépourvus de crêtes. Quant à Aspidiscus montgrinensis SOLÉ (BATTALER 1937), elle montre des crêtes courtes et des monticules dispersés. Ces derniers sont plus nombreux chez Aspidiscus franchii ZUFFARDI-COMERCI, 1921, et s'allongent aux dépens des crêtes. D'autant plus que la face intérieure d'Aspidiscus franchii est parfois trouée, et l'holothèque érodée par endroits, laissant voir les côtes. Sur certains spécimens, en raison de l'érosion des plis concentriques de la face intérieure, une structure de monticule ovale se dégage. Les monticules de la face supérieure représentent le stade initial de bordure de crête. Il est à noter qu'aucune différence entre les crêtes ne se manifeste entre les Latomeandridae et le genre Aspidiscus. Une étude intéressante de la variabilité dans la disposition des crêtes, des monticules et des calices chez Aspidiscus cristatus provenant de plusieurs localités a été réalisée par GILL et LAFUSTE (1987). Nos spécimens montrent une longue crête médiane qui divise le dôme central en deux parties d'égale dimension. Par ce caractère, Ces spécimens sont semblables aux spécimens décrits et figurés par LAMARCK (1801), sous le nom d'Aspidiscus cristatus.

Sur le plan morphologique, on peut signaler quelques différences mineures entre les spécimens d'Aspidiscus cristatus, qui consistent en : (1) une légère différence dans taille des spécimens de la région de Batna; (2) par compa-raison avec les spécimens des autres régions du domaine téthysien, les spécimens de la région de Batna montrent une taille plus petite, une forme plus arrondie, et un nombre de septes inférieur à celui des spécimens d'Égypte et de Palestine, mais ont des tailles et un nombre de septes supérieurs à ceux des spécimens d'Europe de l'Ouest (Tabl. 3). 
Tableau 3 : Dimensions moyennes des spécimens d'Aspidiscus cristatus de quelques régions périméditerranéennes.

\begin{tabular}{ccccccccc} 
Pays \Critère & D & H & Ns/mm & Forme & Bioérosion & Incrustation \\
\hline Algérie & $35-42$ & $12-14$ & $6-7$ & Arrondie & $+/-$ & - \\
Égypte & $53-56$ & 29 & $7-8$ & Allongée & + & + \\
Israël & 50 & 25 & - & Allongée & + & + \\
France & $30-40$ & - & - & Arrondie & - & - \\
Albanie & 34 & - & - & Arrondie & $+/-$ & -
\end{tabular}

La répartition d'Aspidiscus cristatus au sein du domaine téthysien reflète les gradients thermiques autour de l'océan téthysien, certainement en rapport avec les conditions climatiques. En effet, les différences de taille des spécimens de ce taxon sont étroitement liées à la température, la profondeur, la turbidité (indirectement le taux de sédimentation) et l'agitation des eaux à l'interface eau-sédiments.

Il est certain que l'époque cénomanienne a connu un climat globalement chaud, avec des régions polaires sans calottes glaciaires permanentes, mais surtout un gradient thermique entre l'équateur et les pôles beaucoup moins important qu'il ne l'est dans l'actuel. Ce dernier facteur était essentiel dans le contrôle de la répartition spatiale d'Aspidiscus cristatus. Celleci semblait privilégier des eaux chaudes et peu profondes. Ce polypier cénomanien a donc foisonné sur la marge méridionale de la Téthys, avec une bio-érosion très courante affectant la majorité des spécimens de grande taille, tandis que sur la marge septentrionale, les eaux plus froides ont limité son extension vers le nord. Ceci explique le nombre réduit de spécimens connus en Europe de l'Ouest et l'absence totale de ce taxon plus à l'est.

D'après PANDEY et al. (2011), l'augmentation du nombre de septes et la bio-érosion des épiet endobiontes, évoquent un milieu marin de faible profondeur, à énergie hydrodynamique relativement forte et à faible taux de sédimentation.

\section{Conclusions}

La riche collection de spécimens d'Aspidiscus cristatus récoltés dans plusieurs sites de la région de Batna, nous a permis de préciser la répartition spatiale et l'extension stratigraphique de ce taxon, qui est limité au Cénomanien moyen, avec une forte abondance dans la Zone à Acanthoceras cf. rhotomagense. Cela nous permet de définir pour la région étudiée, une zone d'abondance à Aspidiscus cristatus, marqueur du sous-étage Cénomanien moyen pro parte. L'analyse biométrique de plusieurs spécimens de ces scléractiniaires fossiles a permis de mettre en évidence au sein de la population étudiée des variations morphologiques (taille, microarchitectures, ...) en rapport avec les conditions environnementales. L'étude paléontologique et les comparaisons avec les spécimens d'autres régions du pourtour méditerranéen a permis d'éclaircir les conditions paléo-environnementales des milieux de vie de ces organismes, qui semblent tolérer des milieux marins peu profonds, aux eaux relativement chaudes et à substrat vaseux.

\section{Remerciements}

Les auteurs tiennent à remercier les Dr. Gérard BRETON (Le Havre, France), Dr Mohamed BenzaGGAGH (Université Moulay Ismaïl, Meknès, Maroc), un rapporteur anonyme, pour leurs conseils avisés et leurs remarques constructives sur une version préliminaire du manuscrit, et le Dr. Tomáš Kočí (Národní Muzeum, Prague, République tchèque) pour l'identification des tubes de serpules.

\section{Références bibliographiques}

Abdel-Gawad G.I. \& Gameil M. (1995).- Cretaceous and Palaeocene coral faunas in Egypt and Greece.- Coral Research Bulletin, Dresden, vol. 4, p. 1-36.

AlLoiteAu J. (1952).- Madréporaires post-paléozoïques. In : Piveteau J. (éd.), Traité de paléontologie.- Masson, Paris, vol. I, p. 539-684.

Aouissi R., Salmi-Laouar S. \& Ferré B. (2018).Macro-invertébrés du Cénomanien du Djebel Metrassi (Batna, NE Algérie) : Systématique et biostratigraphie.- Estudios geologicos, Madrid, vol. 74, no 2, e082, 39 p.

AvNimeleCH M. (1947).- A new species of Aspidiscus from the Middle Cretaceous of Sinai and remarks on this genus in general.- Eclogae geologicae Helvetiae, Bâle, vol. 40, p. 294-298.

AYOUB-HANNAA W.S. (2011).- Taxonomy and palaeoecology of the Cenomanian-Turonian macroinvertebrates from eastern Sinai, Egypt.- Doctoral Thesis, Bayerischen Julius-Maximilians-Universität Würzburg, $386 \mathrm{p}$.

BARON-SzABO R.C. (2002).- Scleractinian corals of the Cretaceous.- privately published, Knoxville, Tennessee, $539 \mathrm{p}$.

BATTALER J.R. (1937).- La fauna Corallina del Cretacic de Catalunya i regions limitrofes.- Arxius de I'Escuela superior d'Agricultura, Barcelona, vol. 3, p. 1-310.

BELLION Y. (1972).- Étude géologique et hydrogéologique de la partie occidentale des Monts de Bellezma.- Thèse 3ème cycle, Université Paris 6, $186 \mathrm{p}$. 
BenkHerouf F. (1988).- Les foraminifères cénomaniens des Alpes-Maritimes (Sud-Est de la France) et du Djebel Dyr (NE Algérie) : Biostratigraphie et paléo-environnement.- Thèse Doctorat, Université de Nice, no 9, p. 8, 121,173

BUREAU D. (1975).- Esquisse géologique des Monts de Belezma (Aurès, Algérie).- Publication du Service géologique de I'Algérie (Nouvelle Série), Bulletin, Alger, no 45, p. 75-92.

BUREAU D. (1986).- Approche sédimentaire de la dynamique structurale: Évolution mésozoïque et devenir orogénique de la partie septentrionale du fossé saharien (Sud-Ouest constantinois et Aurès, Algérie).- Thèse Doctorat ès Sciences, Université Pierre et Marie Curie, Paris, 2 vols., 441 p.

Burollet P.F. (1956).- Contribution à l'étude stratigraphique de la Tunisie centrale.- $A n$ nales des Mines et de Géologie, Tunis, vol. $18,352 \mathrm{p}$

CoquAND H. (1862).- Géologie et paléontologie de la région Sud de la province de Constantine.- Mémoires de la Société d'Émulation de la Provence, Marseille, vol. II, 341 p.

De Angelis d'OSSAT G. (1901).- Sur une forme singulière d'une colonie d'Aspidiscus cristatus Koenig.- La feuille des jeunes naturalistes, Paris, (4), vol. 31 , no 372 , p. 276280.

DREGER J. (1892).- Ueber einige Versteinerungen der Kreide- und Tertiär- Formation von Corcha in Albanien.- Jahrbuch der Kaiserlich-Königlichen Geologischen Reichsanstalt, Vienne, vol. 42, p. 337-340.

FROMENTEL E. (1877).- Zoophytes, terrains crétacés (10). In : Orbigny A. d' (éd.), Paléontologie Française.- Masson, Paris, vol. 8, p. 433-480 (Pls. 109-120).

GILL G.A \& CHIKHI F. (1991).- Remarks on new occurrences of Aspidiscus, a Cenomanian scleractinian coral, in the Persian Gulf and in Algeria.- Lethaia, Oslo, vol. 24, p. 349-350.

GiLL G.A. \& LAFUSTE J. (1987).- Structure, répartition et signification paléogéographique d'Aspidiscus, hexacoralliaire cénomanien de la Téthys.- Bulletin de la Société géologique de France ( $8 \mathrm{e}$ série), Paris, t. III, $\mathrm{n}^{\circ} 5, \mathrm{p}$. 921-934

GoldFuss A. (1831).- Petrefacta Germaniae tam ea, quae in museo Universitatis Regiae Borussicae Fredericae Wilhelmiae Rhenanae servantur quam alia quaecunque in Museis Hoeninghausiano Muensteriano aliisque extant Iconibus et Descriptionibus Illustrata.- Erster Theil. Arnz \& Co., Düsseldorf, $252 \mathrm{p}$.

GUIRAUD R. (1973).- Évolution post-triasique de l'avant-pays de la chaîne alpine en Algérie, d'après l'étude du bassin du Hodna et des régions voisines.- Thèse Doctorat ès-Scien- ces, Université de Nice, 270 p.

HeRKAT M. (2005).- Analyse quantitative des bioassociations et caractérisation des cortèges sédimentaires et des ensembles paléogéographiques des séries du Cénomano-Turonien des Aurès et du Tell oriental (Algérie).- Bulletin de la Société géologique de France, Paris, t. 176, $\mathrm{n}^{0} 2$, p. 183-190.

HeRKAT M. (2007).- Application of correspondance analysis to paleobathymetric reconstitution of Cenomanian and Turonian (Cretaceous) rocks of Eastern Algeria.- Palæogeography, Palæaeoclimatology, Palæoecology, vol. 254, no 3, p. 583605.

HUBbARD J.A.E.B. (1973).- Sediment-shifting experiments: a guide to functional behaviour in colonial corals. In : BOARDMAN R.S., CHEETHAN A.H., Coates A.G., Oliver W.A. \& Bayer F.M. (éds.), Animal colonies.- Dowden, Hutchinson \& Ross, Stroudsburg, p. 31-42.

HUBBARD J.A.E.B. \& Pocock Y.K. (1972).- Sediment rejection by recent scleractinian corals: a key to palaeo-environmental reconstruction.- Geologische Rundschau, Leipzig, vol. 61, p. 598-626.

KELLY S.R.A. \& Bromley R.G. (1984).- Ichnological nomenclature of clavate borings.- Palaeontology, London, vol. 27, p. 793-807.

KöNIG C.D.E. (1825).- Icones fossilium sectiles.London, 4 p. (8 Pls.).

LAFFITE R. (1939).- Étude géologique de l'Aurès.Thèse de Doctorat ès-Sciences Paris, Bulletin du Service de la Carte Géologique de l'Algérie (1ère série), Alger, vol. 15, 484 p. (1 carte au $1 / 200.000^{\mathrm{e}}$ h.t. ).

LAMARCK J.B.P. (1801).- Système des animaux sans vertèbres.- Deterville, Paris, $432 \mathrm{p}$.

LAMOUROUX J.V.F. (1821).- Exposition méthodique des genres de l'ordre des polypiers.- Agasse, Paris, $115 \mathrm{p}$.

LAPPARENT A. de (1906).-- Traité de géologie (5e édition).- Masson, Paris, 3 vol. : I, 591 p. ; II, p. 595-1237 p. ; III, p. 1241-1911.

MiLNE-EdWARDS H. \& HAime J. (1849).- Recherches sur les polypiers. Mémoire 4, Monographie des Astérides.- Annales des Sciences Naturelles (Série 3), Paris, vol. 10, p. 209-320.

PANDEY D.K., Fürsich F.T., Heinze M., Gameil M. \& Ayoub-HanNaA W.S. (2011).- Aspidiscus cristatus (LAMARCK) from the Cenomanian sediments of Wadi Quseib, east Sinai, Egypt.- Journal of the Paleontological Society of India, Lucknow, vol. 56 , no 1 , p. 29-37.

RENZ C. (1930).- Neue mittelkretazische Fossilvorkommen in Griechenland.- Mémoires de la Société paléontologique Suisse (Abhandlungen der Schweizerischen Paläontologischen Gesellschaft), Bâle, vol. 49, no 5, p. 1-10.

SAVORNin J. (1920).- Étude géologique de la région du Hodna et du plateau sétifien.- Thèse de Doctorat ès Sciences, Bulletin du Service de la Carte géologique de l'Algérie (2ème série), Alger, vol. 7, 502 p. (1 carte 1/800.000). 
Schlosser M. (1924).- Die Cenomanfauna der Bayerischen Alpen.- Centralblatt fur Mineralogie, Geologie und Paläontologie, Stuttgart, vol. 3, p. 82-95.

Shaw M.D. (1738).- Voyages de Mr Shaw M.D. dans plusieurs provinces de la Barbarie et du Levant [Traduction, 1743].- Jean Neaulme, La Haye, 2 vols. (XLIV-414, IV-192), 172 p.

SLAMI R. (2014).- Biostratigraphie et paléoenvironnement de la limite cénomano-turonienne de la région de Batna, à partir de l'étude de microfaune.- Thèse de Magistère, Université de Batna, 229 p.

Slami R., Salmi-laouar S., Ferré B., Aouissi R. \& BENKHEROUF-KECHID F. (2018).- Biostratigraphie, géochimie et réponse des composantes microfauniques aux variations environnementales au passage Cénomanien-Turonien à Thénièt El Manchar (Monts de Bellezma, Batna, NE Algérie).- Estudios Geológicos, Madrid, vol. 74, no 1, e077, 20 p.

SöHLE U. (1897).- Geologische Aufnahme des Labergebirges bei Oberammergau mit besonderer berucksichtigung des Cenomans in den Bayerischen Alpen.- Geognostische Jahreshefte, München, vol. 9, p. 1-66.

THOMAS H. \& OMARA S. (1957).- The Cenomanian compound coral, Aspidiscus cristatus (LAMARCK), from Nezzazat, western Sinai.- Geological Magazine, London, vol. 94, p. 151155.

VoIGT E. (1975).- Tunnelbaue rezenter und fossilier Phoronidea.- Paläontologisches Zeitschrift, Stuttgart, vol. 49, p. 135-167.
VILA J.-M. (1980).- La chaîne alpine d'Algérie orientale et des confins algéro-tunisiens.- Thèse de Doctorat ès Sciences, Université Pierre et Marie Curie, Paris VI, 2 tomes, $665 \mathrm{p}$.

ViVière J.-L. (1985).- Les ostracodes du Crétacé supérieur (Vraconien à Campanien basal) de la région de Tébessa (Algérie du Nord-Est) : Stratigraphie, paléoécologie, systématique.- Thèse de Doctorat 3ème cycle, Université Pierre et Marie Curie, Paris VI, $261 \mathrm{p}$.

WiJSMAN-Best M. (1972).- Systematics and ecology of new Caledonian Faviinae (Coelentrata - Scleractinia).- Bijdragen tot de Dierkunde, Leiden, vol. 42, p. 1-90.

WIJSMAN-BEST M. (1974).- Habitat-induced modification of reef corals (Faviidae) and its consequences for taxonomy.- Proceedings of the 2nd International Coral Reef Symposium, 2, Great Barrier Reef Committee, Brisbane, p. 217-228.

Wilson M.A., Vinn O. \& PALMER T.J. (2014).- Bivalve borings, bioclaustrations and symbiosis in corals from the Upper Cretaceous (Cenomanian) of southern Israel.- Palæogeography, Palæoclimatology, Palæoecology, vol. 414, p. 243-245.

Yahiaoui A. (1990).- La partie inférieure de la série marno-calcaire du Crétacé supérieur (Cénomanien supérieur à Coniacien inférieur) entre Batna et el Kantara (Algérie orientale).- Thèse de Doctorat d'Université, Université de Nancy I, $207 \mathrm{p}$.

ZufFARdi-Comerci R. (1921).- Coelenterati del NeoCretacico della Tripolitania.- Memorie descrittive della Carta geologica d'Italia, Roma, vol. 8, no 1, p. 1-23. 


\section{Planches}

Planche I : 1-8. Différentes vues de quelques spécimens d'Aspidiscus cristatus: 1 (Ba:574), 2 (TM:161), $3($ Me:130), $4(\mathrm{Ba}: 581), 5(\mathrm{Hm}$ 312), $8(\mathrm{~Tb} 297) ; 1 \mathrm{a}, 2 \mathrm{a}, 3 \mathrm{a}, 4 \mathrm{a}, 8 \mathrm{a}$. Face supérieure convexe à contour circulaire ; $1 b, 2 b, 3 b$. Face inférieure plate; 4b, 5, 8b: Face inférieure concave ; 6, 7. Cliché figurant la nature hydnophoroïde : (Mr) Monticule radial, (CC) Centre de calice, (Mm) Monticule médian au nombre de 6 par $\mathrm{mm}$ en moyenne, (Mc) Monticule conique ; 8a. Couronne périphérique ; 8b. Face inférieure couverte d'une holothèque mince avec des crêtes et des plis concentriques. 

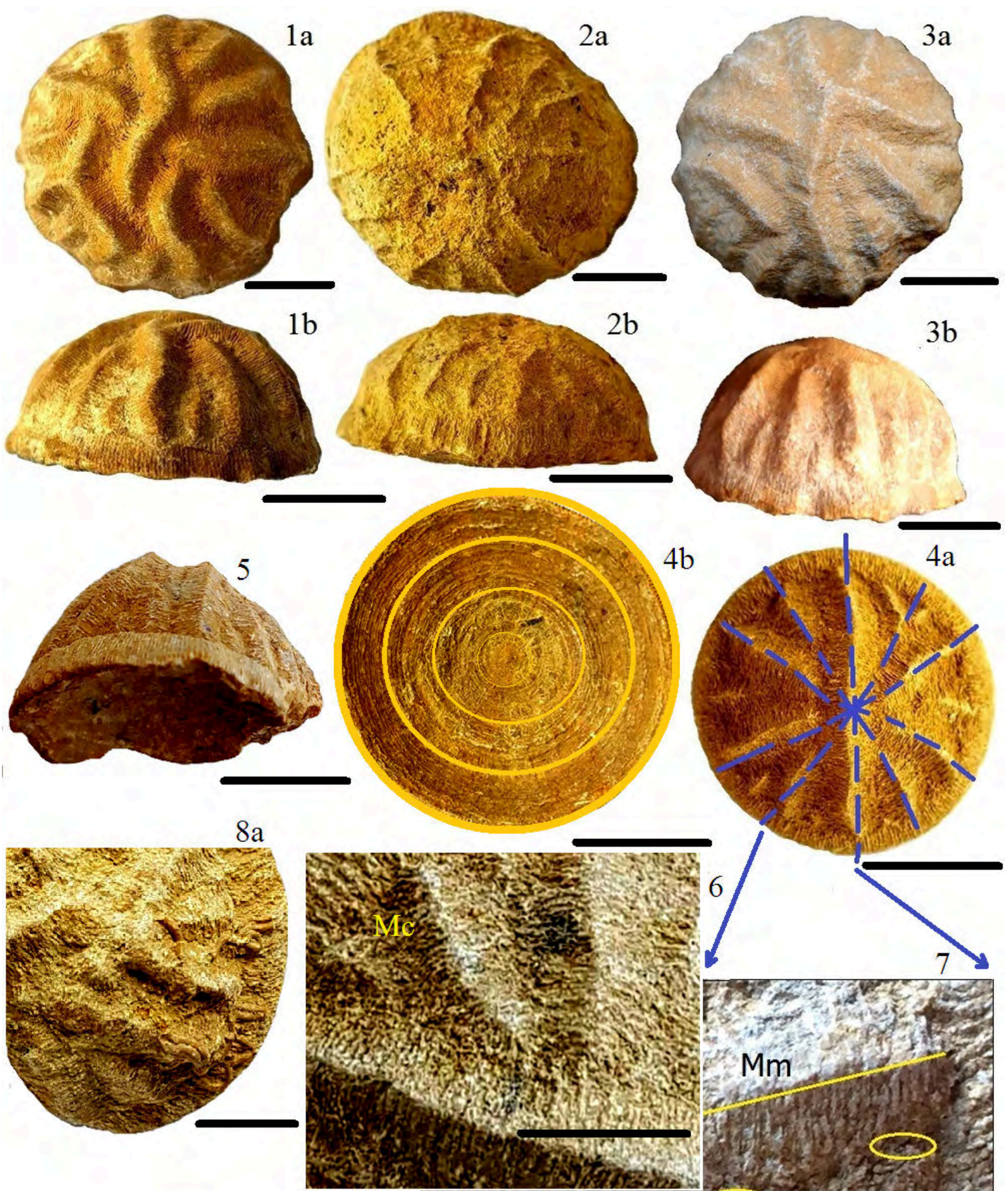

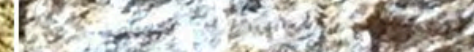
2.2.

$\mathrm{Mm}$

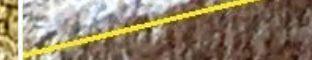
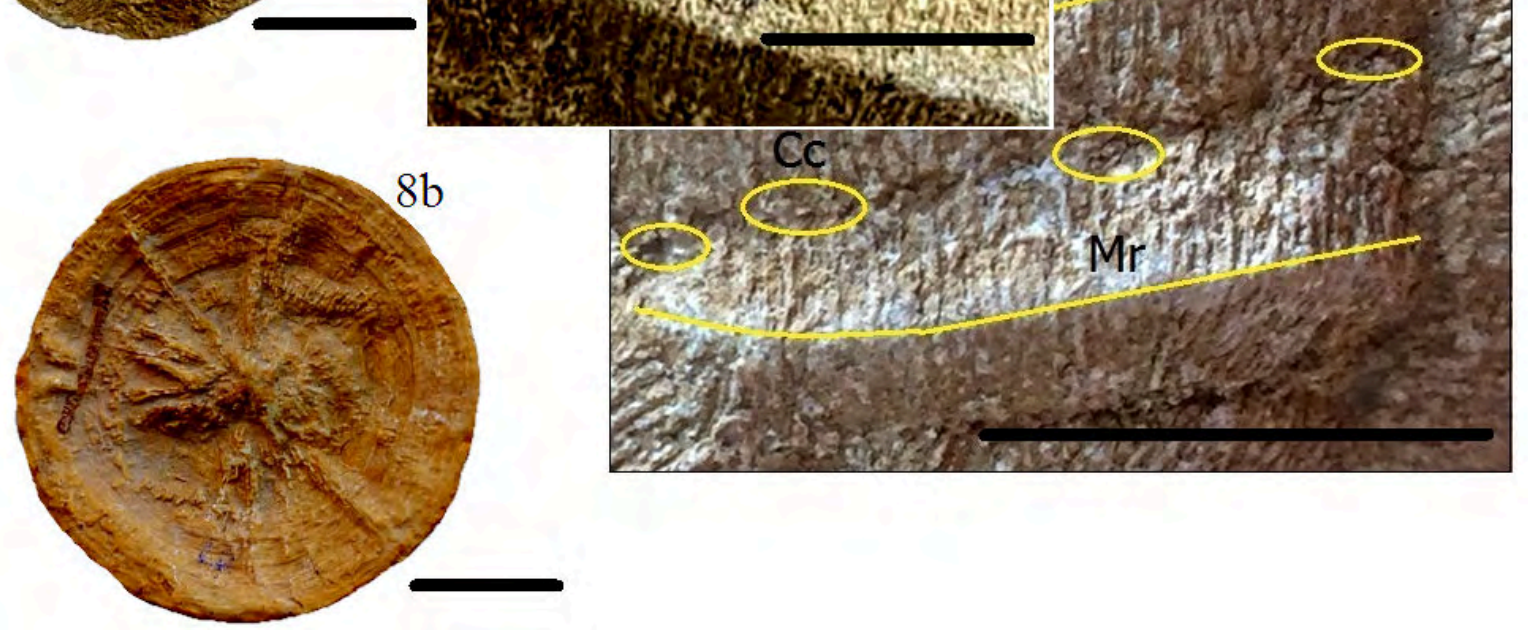
Planche II : 1. Face supérieure à médiane incurvée; 2. Face supérieure à médiane sinueuse ; 1-7. Différentes clichés de spécimens d'Aspidiscus cristatus affectés par des traces bio-érosives ; 1, 2, 4, 5, 6. Aspidiscus cristatus affecté par le genre Gastrochaenolites ; 2b. Aspidiscus cristatus à traces de Gastrochaenolites ampullatus (Kelly \& BROMLEY, 1984) ; 3. Aspidiscus cristatus à traces de Maeandropolydora ichnosp ; 7a, 7b. Aspidiscus cristatus à traces de Glomerula serpentina (GoLDFUSS, 1831). 

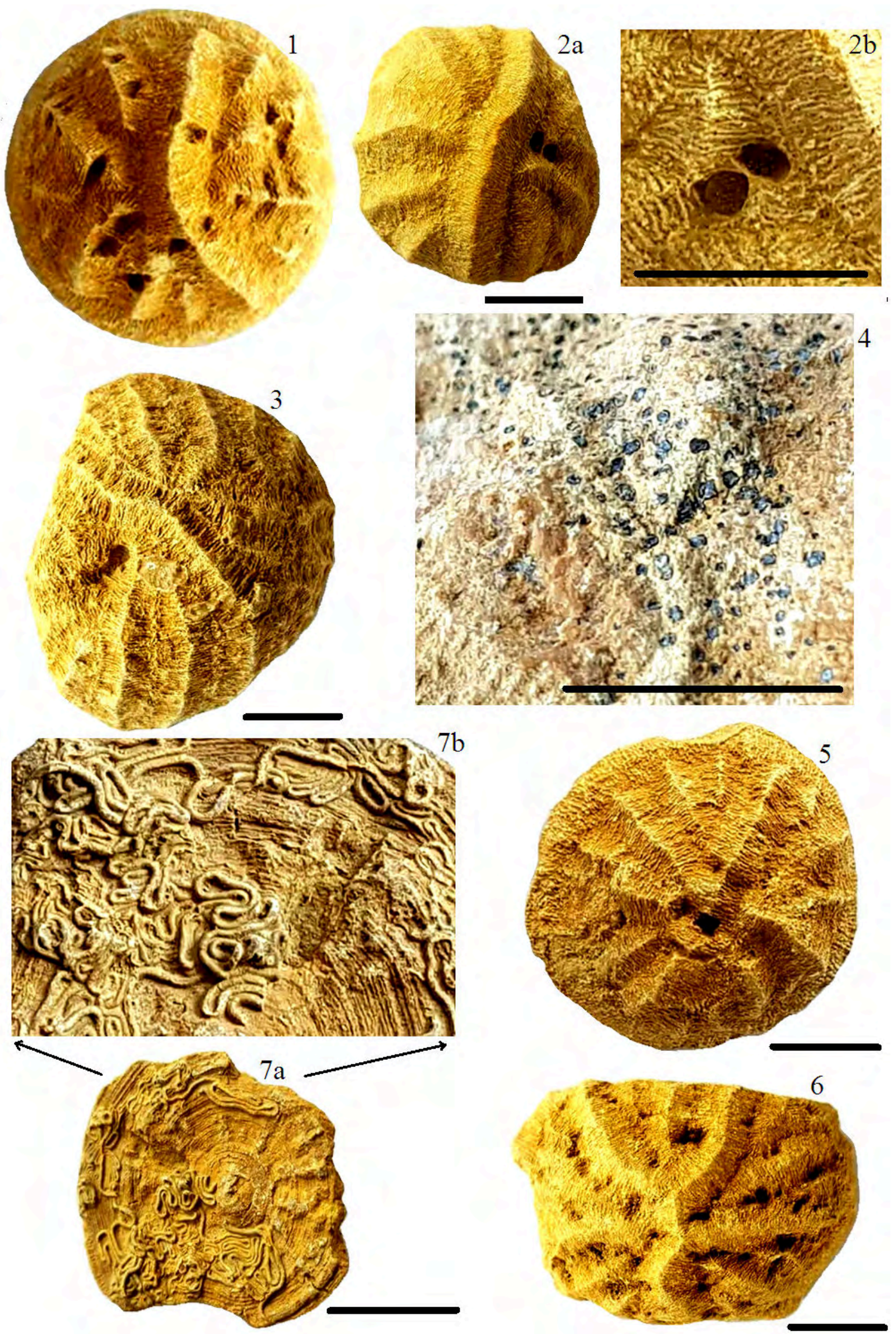